International Journal of Wireless \& Mobile Networks (IJWMN) Vol. 4, No. 3, June 2012

\title{
ROBUST NETWORK FEC-EMBEDDED VIDEO TrANSMISSION OVER ERROR-PRONE WIRELESS CHANNELS
}

\author{
Ghaida Al Suhail ${ }^{1}$ and Sarah A. Subber ${ }^{2}$ \\ Department of Computer Engineering, University of Basrah, Basrah, IRAQ \\ 1'ghaid_alsuhail@yahoo.com ${ }^{2}$ sarah_subberidsco@hotmail.com
}

\begin{abstract}
Most wireless networks incline to include FEC code (Forward error correction) to recover the corrupted packets without retransmission. The sequential packets transmitted over wireless channel are likely to be infected with various errors like noise or deep fading. In this paper, therefore, a frame-level FEC is added across packets at the application layer to define two analytical packet loss models. At the network layer level, Gilbert Elliot Model (GEM) and Extended Gilbert Model (EGM) are considered to characterize the MPEG-4 packets loss over the bursty error-prone wireless channels. The resultant video quality in terms of playable frame rate (PFR) is estimated at the client end when using the underlying TCP-Friendly protocol. Numerical results point out that the EGM-FEC model introduces a robust measure in estimating the perceived video quality in particular at higher packet loss rate and lower effect of packet correlation. Moreover, it is also found that the GEM-FEC model outperforms EGM-FEC but when ignoring the effect of packet correlation.
\end{abstract}

\section{KEYWORDS}

FEC, Gilbert Model, Extended Gilbert Model, Video Streaming \& Wireless Network

\section{INTRODUCTION}

Recently, the IP wireless networks, like Universal Mobile Telecommunications Systems (UMTS), High-Speed Uplink/ Downlink Packet Access (HSUPA/HSDPA) and WiMAX technologies are developed for high data rate transmission [1]-[4]. Among these, wireless environment is unreliable, acknowledges the fact that all wireless real-time video transmission introduce distortions into transmitted streaming packets due to channel fading, multi-path, noise, congestion and interference which may cause to transmit useless packets.

Specifically, a packet-loss in such networks is still a crucial issue in estimating the probability of correctly received video packets at the clients. In wired networks (such as the Internet), packets are lost mainly due to buffer overflows (congestion) at the routers, meanwhile in the wireless hop, packets are often lost due to random bit errors caused by channel variations like noise, mobility, and multi-path fading effects. It is found that a small amount of network error / packet loss can have a dramatic impact on the playable frames at client side. Thus robust video transmission in wireless environment is very challenging and it requires more robust schemes to adapt the variations of network and channel conditions [5]-[7].

In fact, the packet-loss process in IP networks needs an accurate mathematical model to describe it for the design and performance analysis of network applications (e.g., real-time applications). One way is to concentrate on the observed packet loss by itself without considering explicitly the causes that lead to such process [8] [9]. Thus, Markov process (chain)

DOI : 10.5121/ijwmn.2012.4303 
based models are classified to describe packet loss. In $k$-th order Markov chain model characterizing a loss process, the loss of every packet is assumed to be dependent only on the loss status of the previous $k$ packet transmissions. The simplest Markov chain model is the case $k=0$, known as the Bernoulli model. In this model, the packet loss probabilities are independent of each other [10].

Another simple Markov chain model is the case $k-1$, known as the Gilbert model [11], which has been widely used to model end-to-end packet loss processes for real-time applications [7][9]. The former model has also been extended to Gilbert-Elliott model [11]. Although these low-complexity Markov chain models have been frequently used to model such processes [9][11], the accuracy of these Markovian models in capturing the correlation characteristics of real-world packet-loss processes needs to be investigated. For example, Sanneck et al. [12] develop a different Markov chain model, called the extended Gilbert model. There are two categories of extended Gilbert models; those which describe reception run-lengths (RRL) and those which describe loss run-lengths (LRL). In our approach, we concentrate on RRL extended Gilbert models which is derived by Wu and Radha [13] to be applied for MPEG-4 video packet loss process over wireless channel.

Moreover, error-control mechanisms like Automatic Repeat reQuest (ARQ), and Forward Error Correction (FEC) [14]-[15] are also widely used to improve the throughput of a wireless network and eventually compensate the network error (i.e., packet loss). Moreover, since the final performance (e.g. optimal achievable throughput) at the transport level which consequently introduces the optimal play-out rate at client end, depends on the FEC code at video server based on the feedback of radio link layer for wireless client, the consistent TCP model is used to capture interactions and simplify the design complexity. Basically there are advantages and disadvantages for each error-control mechanism. ARQ is simple and needs less bandwidth, where sender retransmits the erroneous packet. However, the retransmission incurs large round trip time (RTT) delay to recover lost packets. FEC is more complexity and incurs less RTT where the erroneous packets are corrected with the help of redundant packets. However, this scheme will incur a bandwidth overhead [16][17].

The studies [18]-[21] which use adaptive packet -level FEC code to improve video quality mostly assume a simple Bernoulli model to describe the packet loss effects. Therefore, in this paper, we investigate two packets loss models: Gilbert-Elliot Model (GEM) and Extended Gilbert Model (EGM) using frame-level FEC at the application layer to correct packet loss due to wireless environment conditions. The models employ the underlying TCP-Friendly Rate Control for MPEG-4 video transmission. As a result, the video quality can be estimated in terms of playable frame rate at the client end under various packet-level FEC codes at the application layer. The results obtained introduce a good comparative study for robust network FEC embedded video transmission over a highly correlated packet loss over wireless channel.

The rest of this paper is organized as follows. Section 2 describes several related works on video quality over wireless networks. Section 3 provides background to the work. Our proposed analytical models of MPEG video transmission are presented in Section 4. Results and performance comparison are addressed in Section 5. Finally, Section 6 summarizes the conclusion and outlines some future works

\section{RELATED WORK}

To carry out a high video quality, most wireless networks incline to include FEC code to recover the corrupt packets and avoid retransmissions. Video streaming have strict constrains on delay latency and delay jitter. This makes FEC as an optimal choice for many real-time applications. Many studies therefore have devoted to improve the video quality performance using different strategies. One solution may tackle the problems of how modifying the TCP model [22][23]; and the other is how improving the link reliability observed by TCP [5][6][24]. 
International Journal of Wireless \& Mobile Networks (IJWMN) Vol. 4, No. 3, June 2012

The later solution mainly includes a cross-layer design of hybrid schemes to provide a required quality link reliability over wireless links. There exist many recent cross-layer approaches, for example: adaptive rate control [6], adaptive selective Repeat (ASR) protocols (re-transmission) [24], finite-length queuing at the data link layer coupled with adaptive modulation and coding (AMC) at the physical layer [5], optimal time slot for optimal resource allocation [25], and finally adaptive FEC at packet/byte/bit level (e.g., BCH, RCPC and RS codes) [2][5][21].

On the other hand, many researches [18]-[28] have devoted on video streaming based on TCP/UDP protocols in different wired/wireless IP-packet networks. Specifically, TCP has become popular protocol because of its easy handling and deployment. TCP features in order delivery and reliable end-to-end transport, which makes additional tools like error concealment, unnecessary at the clients. In low-latency networks, TCP introduces good throughput performance and low end-to-end delays, which makes TCP-based interactive services possible. In [28], for example, they propose a client-driven video transmission scheme by utilizing multiple HTTP/TCP streams. In [6], they evaluate video streaming based on TCP-Friendly Rate Control (TFRC) using Bernoulli packet loss model for a single and Multiple TFRC flows in UMTS network.

\section{NetWork PRELIMINARIES}

\subsection{MPEG}

In the last years, several video standards have been developed for 3G mobile multimedia communications like H.263, MPEG-4, and H.264/AVC [9]. MPEG video is one of the most commonly used video compression standard which is encoded into three different types of frames- I (Intra-coded), P (Predictive-coded), and B (Bi-directional Predictive coded) frames. In this standard, I-frame is independently coded, while P-frame is coded based on the prediction of object movement of the previous I or P frame. The B-frame is coded based on the differences between the previous I or $\mathrm{P}$ frame and the next I or $\mathrm{P}$ frame. Thus, there is a certain dependency relationship between I, P, B frames as shown in Figure 1 [11][18].

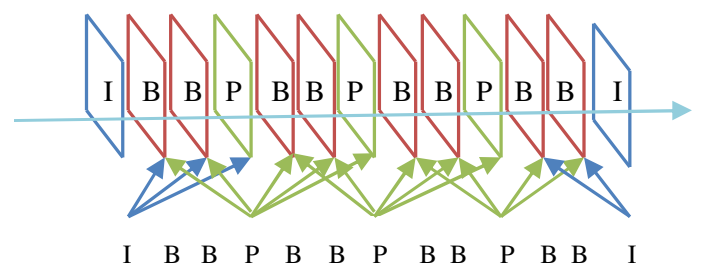

Figure 1.The structure of a GOP and the inter-frame dependency relationship

\subsection{Forward Error Correction (FEC)}

In a lossy channel packets are dropped due to routing disruption, interference, and congestion in the intermediate nodes [4], therefore to protect video packets from losses in a wireless environment, FEC blocks are applied at the application-layer. In [18], Wu et al.'s proposed an analytical model which is known as the frame-level FEC technique. The FEC packets are generated based on individual frames (I, P, or B) as shown in Figure 2.

Thus, when $K$ packets are transmitted with $(N-K)$ redundant FEC packets with packet loss probability $P_{\text {avg }}$, the successfully probability after encoding is defined by a binomial trial; 


$$
P_{\text {suc }}\left(N, K, P_{\text {avg }}\right)=\sum_{i=K}^{N}\left(\begin{array}{l}
N \\
i
\end{array}\right)\left(1-P_{\text {avg }}\right)^{i} \cdot P_{\text {avg }}{ }^{N-i}
$$

\begin{tabular}{|c|}
\hline I -Fame Packets \\
\hline I-Frame FEC Packets \\
\hline P-frame Packets \\
\hline P-Frame FEC Packets \\
\hline B-Frame Packets \\
\hline B-Frame FEC Packets \\
$\ldots \ldots \ldots \ldots \ldots \ldots$ \\
$\ldots \ldots \ldots \ldots$ \\
\hline B-Frame Packets \\
\hline B-Frame FEC Packets \\
\hline
\end{tabular}

Figure 2.Arrangement of source and FEC Packets in frame-level FEC technique.

\subsection{TCP-Friendly Throughput}

We focus on the video traffic controlled by TCP-Friendly protocol over wireless network because of some protocol advantages. It does not cause network instability and avoids congestion collapse. It is fair to TCP flows, which is the dominant source of traffic on the internet, and finally this protocol has lower fluctuation compared to TCP. That makes TCPFriendly more appropriate for real time applications, which requires a constant video quality [3].

In this paper, we consider a video flow in a point-to-point network which is simply composed of one base station (access point) in UMTS and a single user end. This last wireless link is connected to a wired Internet via this base station [3][6]. By adjusting the sending rate to the desirable rate determined by an underlying TCP-Friendly Rate Control (TFRC), one can achieve the required quality of service $(\mathrm{QoS})$ of video applications over a wireless link. Thus the normalised available bandwidth of a TFRC video session with respect to TCP packet size can be expressed as,

$$
T=\frac{S}{t_{R T T} \sqrt{\frac{2 P_{a v g}}{3}}+t_{R T O}\left(3 \sqrt{\frac{3 P_{a v g}}{3}}\right) P_{\text {avg }}(1+32)}
$$

where $S$ is the TCP packet size [byte], $P_{\text {avg }}$ stands for the average packet loss probability, i.e., loss event rate due to only the channel bit errors and there is no buffer overflow effect at the base station. $t_{R T T}$ is the round-trip time [sec], and $t_{R T O}$ is the TCP retransmit time out value [sec].

\subsection{GOP Rate}

As $T$ is TFRC throughput, the GOP rate can be written as; 
$G=\frac{T / S}{\left(S_{I}+S_{I F}\right)+N_{P}\left(S_{P}+S_{P F}\right)+N_{B}\left(S_{B}+S_{B F}\right)}$

$N_{P}$ represents the number of $P$ frame in a GOP and $N_{B}$ is the number of $B$ frame in a GOP.

\subsection{Playable Frame Rate (PFR)}

The total PFR by Wu et al.'s, technique is given by [18];

$$
P F R=G . P_{S I} \cdot\left(1+\frac{P_{S P}-P_{S P}^{N_{P}+1}}{1-P_{S P}}+N_{B P} \cdot P_{S B} \cdot\left(\frac{P_{S P}-P_{S P}^{N_{P}+1}}{1-P_{S P}}+P_{S I} \cdot P_{S P}^{N_{P}}\right)\right)
$$

Where $P_{S I}, P_{S P}$ and $P_{S B}$ are the probabilities of success transmission of I, P, or B frame.

\section{THE PROPOSED MODEL}

\subsection{GEM-FEC Video Model}

Recently many studies used the measurements of burst error over a wireless channel by the well known Gilbert Elliot model (GEM) [8][13]. In the present work we further assume GEM as virtual channel with two nodes, to improve the video quality by estimating the playable frame rate.

The state diagram of the model is shown in Figure 3, the model represents two states; the good state (good packet $P_{0}$ ) and the bad state (bad packet $P_{1}$ ).

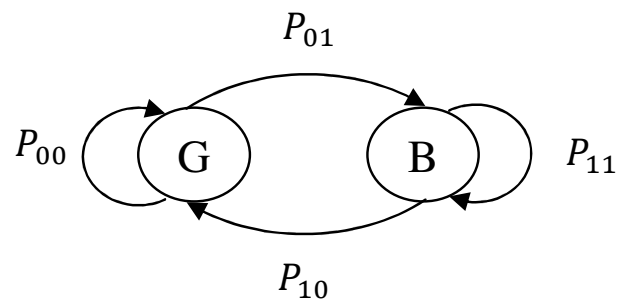

Figure 3.Gilbert Elliot state diagram for packet level

The $P_{01}$ is the state probability for transition form good to bad, and $P_{10}$ is the state probability from transition from bad to good . The $\pi_{1}$ and $\pi_{0}$ study state probability being in state $P_{0}$ and $P_{1}$ and $\pi_{1}=\frac{P_{01}}{P_{01}+P_{10}}, \pi_{0}=\frac{P_{01}}{P_{01}+P_{01}}$ respectively. The average packet loss rate product by the GE model is defined as

$$
P_{\text {avg }}=P_{0} \pi_{0}+P_{1} \pi_{1}
$$

The GEM is memory less model, where Packet error is produced by a sequence of independent trial. Each packet has $P_{a v g}$ being flipped and $1-P_{a v g}$ being successfully transmitted, $P_{a v g}$ is then the packet loss probability for the wireless channel. 
With a specific I, P, and B frame sizes and number of FEC blocks, (1) can be used to compute the probabilities of successful transmission for each frame type as following;

$$
\begin{aligned}
& G E M P_{S_{I}}=\sum_{i=S_{I}}^{S_{I}+S_{I F}}\left(\begin{array}{l}
S_{I}+S_{I F} \\
i
\end{array}\right)\left(1-P_{a v g}\right)^{i} P_{a v g}^{S_{I}+S_{I F}} \\
& G E M P_{S_{I}}=\sum_{i=S_{P}}^{S_{P}+S_{P F}}\left(\begin{array}{l}
S_{P}+S_{P F} \\
i
\end{array}\right)\left(1-P_{a v g}\right)^{i} P_{a v g}^{S_{P}+S_{P F}} \\
& G E M P_{S_{I}}=\sum_{i=S_{B}}^{S_{B}+S_{B F}}\left(\begin{array}{l}
S_{B}+S_{B F} \\
i
\end{array}\right)\left(1-P_{a v g}\right)^{i} P_{a v g}^{S_{B}+S_{B F}}
\end{aligned}
$$

The total PFR using GEM-FEC is expressed as

$$
\begin{gathered}
P_{G E R_{-F E C}}=G \cdot G E M P_{S I} \cdot\left(1+\frac{G E M P_{S P}-G E M P_{S P}{ }_{S P}+1}{1-G E M P_{S P}}\right. \\
+N_{B P} \cdot G E M P_{S B} \cdot\left(\frac{G E M P_{S P}-G E M P_{S P}^{N_{P}+1}}{1-G E M P_{S P}}+\right. \\
\left.\left.G E M P_{S I} \cdot G E M P_{S P}^{N_{P}}\right)\right)
\end{gathered}
$$

\subsection{EGM-FEC Video Model}

The Second proposed model for packet loss probability is a model which was derived by $\mathrm{Wu}$ and Radha [13] have been used, where the authors extends the two-state Gilbert model using the number of correctly received packets as a indexes for Gilbert states

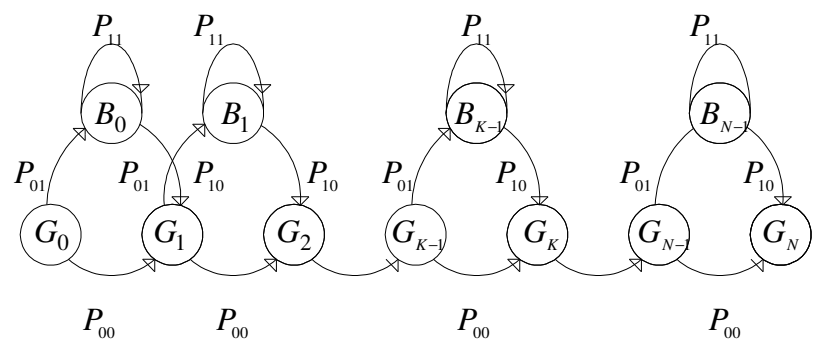

Figure4. The state transition diagram of Extended Gilbert Model (EGM).

The Extended Gilbert model is build on the probability of receiving correctly $K$ packets among $N$ packets transmitted over Gilbert is giving by;

$$
\phi(N, K)=\pi_{0}\left(\phi_{G_{0} G_{K}}(N)+\phi_{G_{0} B_{K}}(N)\right)+\pi_{1}\left(\phi_{B_{0} G_{K}}(N)+\phi_{B_{0} B_{K}}(N)\right)
$$

Where 


$$
\begin{aligned}
& \phi_{G_{0} G_{K}}(N)= \\
& \begin{cases}\sum_{m=1}^{K}\left(\begin{array}{l}
K \\
m
\end{array}\right)\left(\begin{array}{l}
N-K-1 \\
m-1
\end{array}\right) P_{01}{ }^{m} P_{10}{ }^{m} P_{00}{ }^{K-m} P_{11}{ }^{N-K-m} 0<K<N \\
0 & K=0 \\
P_{00}{ }^{N} & K=N\end{cases} \\
& \begin{array}{l}
\phi_{G_{0} B_{K}}(N)= \\
\left\{\begin{array}{l}
\sum_{m=0}^{K}\left(\begin{array}{l}
K \\
m
\end{array}\right)\left(\begin{array}{l}
N-K-1 \\
m-1
\end{array}\right) P_{01}{ }^{m+1} P_{10}{ }^{m} P_{00}{ }^{K-m} P_{11}{ }^{N-K-m-1} 0<K<N \\
0
\end{array}\right.
\end{array} \\
& \phi_{B_{0} G_{K}}(N)= \\
& \left\{\begin{array}{l}
\sum_{m=1}^{K-1}\left(\begin{array}{l}
K-1 \\
m
\end{array}\right)\left(\begin{array}{l}
N-K \\
m
\end{array}\right) P_{01}{ }^{m} P_{10}{ }^{m+1} P_{00}{ }^{K-m-1} P_{11}{ }^{N-K-m-1} 0<K<N \\
0
\end{array}\right. \\
& \phi_{B_{0} G_{K}}(N)=
\end{aligned}
$$

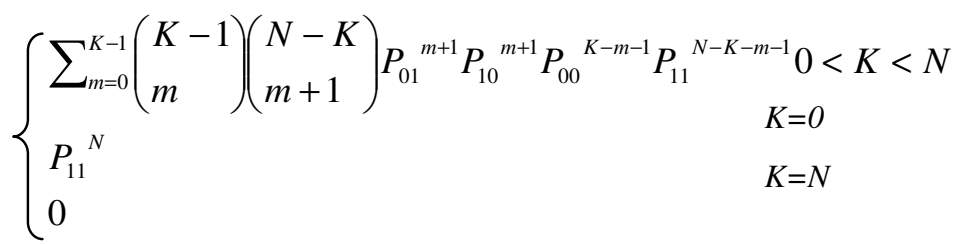

The state probability $P_{01}$ and $P_{10}$ (or $P_{00}=1-P_{01}$ and $P_{11}=1-P_{10}$ ) are the effective parameters on the evaluation of $\phi(N, K)$. More useful parameters can be involved to improve the performance. In [13], the authors used the average loss rate $P_{\text {avg }}$, and the packet correlation $\boldsymbol{\rho}$, to give another perception to the state transition probabilities.

$$
\begin{aligned}
& P_{01}=P_{\text {avg }}(1-\rho) \\
& P_{10}=\left(1-P_{\text {avg }}\right)(1-\rho)
\end{aligned}
$$

For FEC codes, the probability that the node can receive enough packets to decode an FEC block when $\mathrm{K}$ packet is transmitted is;

$$
P_{\text {suc }}=\sum_{i=K}^{N} \phi(N, K)
$$

According to (17), the computation of the successful transmission probabilities for a specific $I$, $P$, and $B$ frame sizes and FEC block can be written as;

$$
E G M P_{S_{I}}=\phi\left(S_{I}+S_{I F}, S_{I}\right)
$$




$$
\begin{aligned}
& E G M P_{S_{P}}=\phi\left(S_{P}+S_{P F}, S_{P}\right) \\
& E G M P_{S_{B}}=\phi\left(S_{B}+S_{B F}, S_{B}\right)
\end{aligned}
$$

Then the total PFR using EGM-FEC can be written as;

$$
\begin{aligned}
P F R_{E G M-F E C}= & G \cdot E G M P_{S I} \cdot\left(1+\frac{E G M P_{S P}-E G M P_{S P}{ }^{N_{P}+1}}{1-E G M P_{S P}}+N_{B P} \cdot E G M P_{S B} .\right. \\
& \left.\left(\frac{E G M P_{S P}-E G M P_{S P}{ }^{N_{P}+1}}{1-E G M P_{S P}}+E G M P_{S I} \cdot E G M P_{S P}{ }^{N_{P}}\right)\right)
\end{aligned}
$$

\section{Simulation ReSUlt}

Based on the above assumptions, we can develop the following illustrative steps to find the optimal playable frame rate using both GEM-FEC and EGM-FEC video models defined in Section 4.

1. In order to compare GEM-FEC and EGM-FEC, one can determine the $P_{\text {avg }}$ from (1) by defining a specified state probability. For each set of other system variables, we can compute the PFR.

2. For GEM-FEC model, assuming specific average loss rate, and packet correlation $\rho$, PFR defined in (9) can be estimated using (6), (7) and (8), respectively. Using the three different cases of FEC weights with a certain probability of packet loss; we can evaluate the robust optimal PFR.

3. For EGM-FEC model, the PFR can be evaluated using (18), (19), (20) and (21) for given packet correlation, FEC weights, and certain loss probability. This allows us to characterize the variation of the PFR under different conditions of packet correlation and FEC weights.

\subsection{System Settings}

Table 1 describes network characteristic of many typical network connection [13][18][26]. A GOP $\left(N_{P}, N_{B P}\right)$ pattern is considered with $N_{P}=2$ and $N_{B P}=3$. In Table 2 a list of three FEC weights is defined to be used in our experiments.

Table 1. Simulation Parameters

\begin{tabular}{|l|c|}
\hline Parameter & value \\
\hline$t_{R T T}, t_{R T O}$ & $168 \mathrm{~ms}, 4 \times t_{R T T}$ \\
\hline$N_{P}, N_{B P}$ & 3,2 \\
\hline$S_{I}$ & $24.64 \mathrm{~KB} \quad[25$ packet $]$ \\
\hline$S_{P}$ & $7.25 \mathrm{~KB} \quad[8$ packet $]$ \\
\hline$S_{B}$ & $2.45 \mathrm{~KB}[3$ packet $]$ \\
\hline$P_{00}, P_{11}$ & $0.96,0.94$ \\
\hline$P_{0}, P_{1}$ & $0.001,0.001$ to 0.1 \\
\hline $\boldsymbol{\rho}$ & 0.9 \\
\hline
\end{tabular}

Table 2. FEC Weights

\begin{tabular}{|c|c|c|c|}
\hline $\begin{array}{c}\text { FEC } \\
\text { weight }\end{array}$ & $S_{I F}$ & $S_{P F}$ & $S_{B F}$ \\
\hline Light & 1 & 1 & 0 \\
\hline Medium & 4 & 2 & 0 \\
\hline High & 8 & 4 & 1 \\
\hline
\end{tabular}




\subsection{Performance Evaluation}

This section describes the PFR of an MPEG4 Video streaming over wireless channel using two packets loss models: the GEM-FEC video model and EGM-FEC video model addressed in Section 4 . The performance evaluation is carried out by considering the parameters in Table 1 for the fact that maximum frame rate allowed over Internet is $30 \mathrm{fps}$. The results are conducted using Matlab package.

Experiment 1: $\quad$ Figure 5 shows the video quality vs. the average packet error rate using GEM-TFRC and EGM-TFRC packet loss models with no FEC weights. We consider there is no packet correlation for GEM-TFRC because it was found through our experimental results that there is no significant effect on video quality when $\rho$ factor varies between 0 and 1 . On the other hand, we evaluated the EGM-TFRC model when $\rho$ factor is considered to be close to 1 . As a result, it is clearly noticed that a highest PFR can be achieved for packet loss probability less than $2 \%$ in both models. However, $\boldsymbol{\rho}$ factor has a slight impact on the resultant video quality at packet loss rates greater than $2 \%$ especially in EGM-TFRC model. In other word, one can conclude that the GEM-FEC becomes closer to the EGM-FEC at packet loss probabilities below $2 \%$.

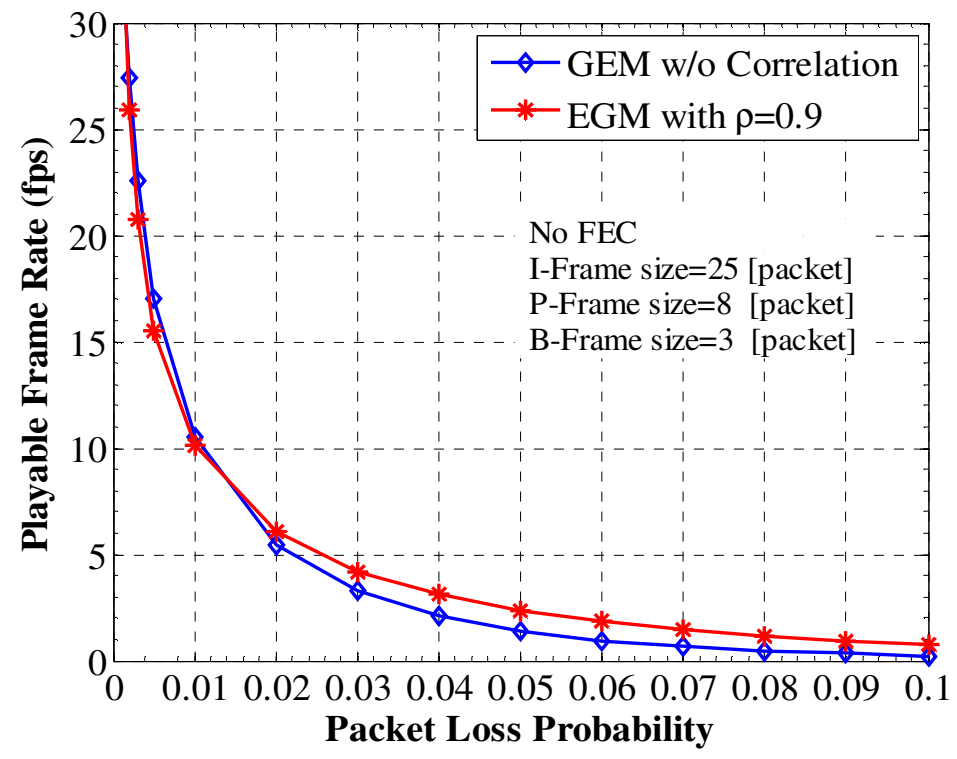

Figure 5.Playable Frame Rate of GEM and EGM models with no FEC 


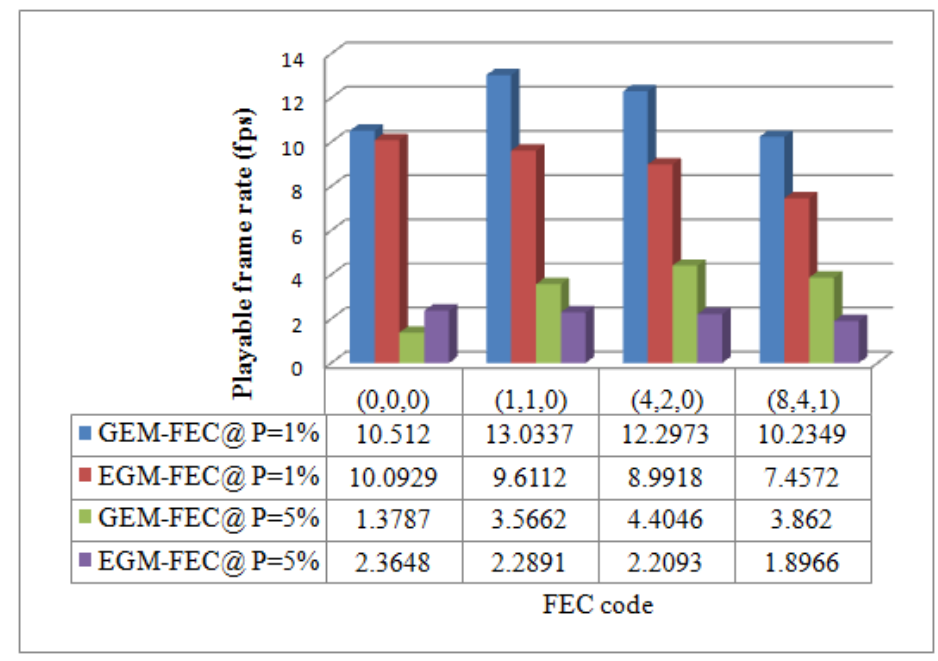

Figure 6.Playable Frame Rate of GEM-FEC and EGM-FEC with correlation factor of 0.9

Experiment 2: The results obtained in Figure 6 depict the PFR variations when we add three different FEC weights to both GEM-FEC and EGM-FEC model, respectively. As mentioned in Fig. 5, only EGM-FEC model is evaluated with correlation factor of 0.9 ; and this factor effect is discarded on the performance of GEM-FEC model. When average loss probability is considered to be $1 \%$ (less than $2 \%$ ), it is clearly observed that GEM-FEC provides highest PFR at light weight FEC $(1,1,0)$ compared to EGM-FEC. Moreover, the former model responds less quality performance at high weight of $\operatorname{FEC}(8,4,1)$. Now, when average loss probability loss increases to be $5 \%$ it is also found that GEM-FEC outperforms EGM-FEC particularly at medium FEC weight $(4,2,0)$.

Experiment 3: In Figure 7, the effect of packet correlation factor is examined for both proposed packet loss models under different FEC weight codes presented in Table 2. Figure 7 (a) shows the resultant PFR when the average loss rate is set to be $1 \%$, and $\rho$ correlation factor is changed from 0.1 to 0.9 for only EGM-FEC model; meanwhile the $\rho$ correlation factor has no significant role in GEM-FEC model (as shown in Fig. 5). Therefore, it is shown that when $\rho$ varies in the range of 0.5 to 0.9 and FEC weight increases, the perceived PFR may clearly decline. In contrary, when $\rho$ decreases to be less than 0.5 (say, 0.1 ), the resultant PFR indicates much better performance on FEC weight $(4,2,0)$.

In Figure 7 (b), the effect of higher average loss rate (say 5\%) has another serious impact on video quality at the client end. From the chart, one can see that when packet correlation varies from 0.5 to 0.9 , the PFR refers to the better performance on FEC weight $(4,2,0)$. However, once the packet correlation decreases to be 0.1 , there is a clear improvement in video quality. The perceived PFR achieves the highest quality especially when FEC weight becomes $(8,4,1)$. Thus, we can conclude that EGM model with high FEC provides a robust measure in video transmission over wireless channel compared to GEM model. 
International Journal of Wireless \& Mobile Networks (IJWMN) Vol. 4, No. 3, June 2012

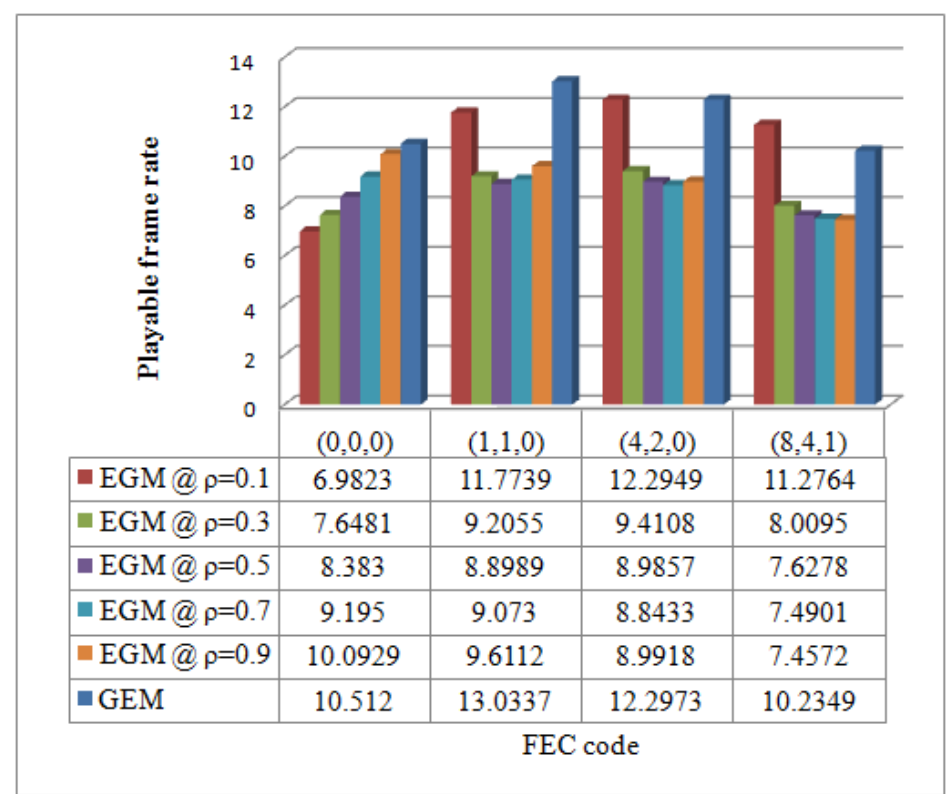

(a)

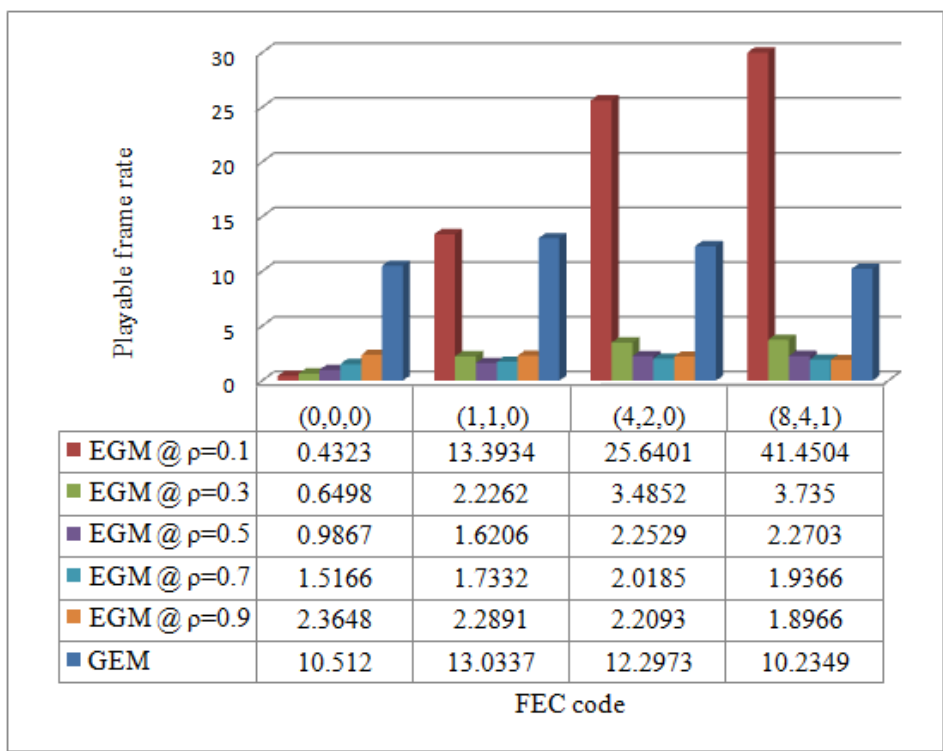

(b)

Figure7.The impact of packet correlation on video quality for two various packet loss rates (a) $P_{\text {avg }}=1 \%$ and (b) $P_{\text {avg }}=5 \% \rho$ 
International Journal of Wireless \& Mobile Networks (IJWMN) Vol. 4, No. 3, June 2012

\section{CONCLUSIONS AND Future WORK}

In this paper, a frame-level FEC at the application layer is considered to define two analytical packet loss models. The models are applied to estimate the video quality over Gilbert wireless channels with a highly correlated error. The results clearly introduce a good comparative performance. The GEM-TFRC video model shows a good performance in high packet loss probability; meanwhile GEM and EGM both show reasonable performance but for average packet loss rate below 2\%. With FEC, it is found that GEM-TFRC at light FEC of $(1,1,0)$ provides the best performance. In contrary, EGM-TFRC with a small packet correlation below 0.5 and a FEC weight of $(4,2,0)$ outperforms GEM-TFRC; otherwise a clear degradation in video quality can be achieved with the increase of packet correlation. As a result, we can conclude that these models are applied to be helpful in predicting the robust video quality over wireless channels. The Future work can be extended to involve the GOP-level FEC and retransmission techniques to improve the performance of video quality under different conditions of wireless environment.

\section{REFERENCES}

[1] H. Holma and A. Toskala, (2006), HSDPA /HSUPA for UMTS High Speed Radio Access for Mobile Communications, John Wiley, England.

[2] Pei Y. and Modestino J. W., (2004) "Interactive Video Coding and Transmission over Heterogeneous Wired-to-Wireless IP Networks using an Edge Proxy", EURASIP Journal on Applied Signals, pp. 253-264.

[3] A. Lo, G. Heijenk and I. Niemegeers, (2005), “ Performance Evaluation of MPEG-4 Video Streaming over UMTS Networks Using an Integrated Tool Environment", Proceedings of the Inter. Symposium on Performance Evaluation of Computer and Telecommunication Systems, pp. 676-682.

[4] G. A. AL-Suhail, M. Fleury and S. M. Al-Majeed, (2011), "Mobile Video Streaming Over Heterogeneous Networks", Book Chapter 13, Innovations in Mobile Multimedia Communications and Applications: New Technologies, Edited by Ismail Khalil and Edgar R. Weipl, IGI Global, pp. 175-200.

[5] Q. Liu, S. Zhou, and G. B. Giannahis, (2005) "Queuing with Adaptive Modulation and Coding over Wireless Links: Cross-Layer Analysis and Design,” IEEE Trans. Wireless Comm., Vol. 4, No. 3.

[6] M. Chen and A. Zakhor, (2005), "Rate Control for Streaming Video over Wireless," IEEE Wireless Communication, pp. 32-41.

[7] A. Khan, L. Sun, E. Ifeachor, J.O. Fajardo, F. Liberal, (2010), "Video Quality Prediction Model For H.264 Video over UMTS Networks and their Application in Mobile Video Streaming", IEEE Communication (ICC).

[8] X. Yu, W. Modestino and X. Tian, (2005), "The Accuracy of Gilberts Models in Predicting Packet-Loss Statistics for Single-Multiplexer Network Model”, IEEE International Conference.

[9] G. Haßlinger and O. Hohlfeld, (2008), "The Gilbert-Elliott Model for Packet Loss in Real Time Services on the Internet", Darmstadt University of Technology, Germany.

[10] E. N. Gilbert, (1960) "Capacity of a Burst Noise Channel”, Bell Sys. Tech. Journal, vol. 39, p. 1253.

[11] Y. Baguda, N. Fisal, S. Syed, S. Yusof, M. Abdullah, and A. Zulkarmawan, (2008), “ Mobile Streaming of H.264 Video over Gilbert-Elliotts Channel ", World Academy of Science, Engineering an Technology 
International Journal of Wireless \& Mobile Networks (IJWMN) Vol. 4, No. 3, June 2012

[12] H. Sanneck, G. Carle, and R. Koodli, (2000) “A Framework Model for Packet Loss Metrics Based on Loss Run Length," Proceedings of SPIE/ACM SIGMM Multimedia Computing and Networking Conference.

[13] M. Wu and H. Radha, (2005) "Network Embedded FEC for Overlay and P2P Multicast over Channels with Memory", Conf. on Information Sciences and Systems, The John Hopkins University.

[14] H. Liu, H. Ma, M. E. Zarki and S. Gupta, (1997), ”Error Control schemes for Networks: An Overview". Mobile Networks and Applications, pp167-182.

[15] P. Paul and S. Barman, (2009) "ARQ Protocols on Mobile Communication and Networks", International Journal of Computer and Electrical Engineering, Vol. 1, No. 2, pp.1793-8163.

[16] H. Han, (2011) "Performance Improvement of TCP Reno Based on Monitoring the Wireless Packet Loss Rate”, Physics \& Electronics Engineering Department, Xiangfan University, China.

[17] M.R.Ebenezar jebarani and T.Jayanth, (2010), "An Analysis of Various Parameters in Wireless Sensor Networks Using Adaptive FEC Technique”, International Journal of Ad hoc, Sensor \& Ubiquitous Computing (IJASUC), Vol.1, No.3.

[18] H. Wu, M. Claypool and R. Kinicki, (2003) "A Model for MPEG with Forward Error Correction and TCP-Friendly Bandwidth", Proceedings of Workshop on Network and Operating Systems Support for Digital Audio and Video (NOSSDAV), Monterey, California, USA.

[19] Y. Yuan, B. Cokburn, T. Sikora, and M. Mandal, (2006), "A GoP Based FEC Technique for Packet Based Video Streaming", Proceedings of the $10^{\text {th }}$ WSEAS International Conf. on Communications, Greece.

[20] R. Razavi, M. Fleury, \& M. Ghanbari (2009), “Adaptive Packet-Level Interleaved FEC for Wireless Priority Encoded Video Streaming”, School of CSEE, University of Essex, Wivenhoe Park, Colchester CO4 3SQ, UK.

[21] G. A. AL-Suhail and R. Kennedy, (2009), "Modelling of Adaptive Wireless Link for MPEG-4 Video Transport in UMTS Network," Inter Conf. Signal processing and communication system ICSPCS, USA.

[22] Akan O. B. and Akyilldiz I. F., "Arc: The Analytical Rate Control Scheme for Real-time Traffic in Wireless Networks," IEEE/ACM Trans. Networking, (12) 4: 634-644, 2004.

[23] H. Oda, H. Hisamatsu, and H. Noborio, (2011) "Design, Implementation And Evaluation of Congestion Control Mechanism for Video Streaming”, International J. of Computer Networks \& Communications (IJCNC), vol. 3, no. 3, .193-204.

[24] Xylomenos G. and Makidis M., "Adaptive Link Layer Protocols for Shared Wireless Links," Proceedings of ACM MOBIMEDIA, 2007

[25] N. M. Aripin, R. A. Rashid, N. Fisal, A.C. Lo, S.H. Ariffin, and S. K. Yusof, (2010), “A CrossLayer Approach in Sensing and Resource Allocation for Multimedia Transmission over Cognitive UWB Networks, Vol. 2010, EURASIP Journal on Wireless Communications and Networking, 10 pages.

[26] C. H. Lin, C. H. Ke and C. K. Shieh, (2004) "A Study of MPEG Video Transmission in Lossy Wireless Networks", Electrical Engineering Department, National Cheng Kung University, Taiwan.

[27] T. Ahmed, A. Mehaoua, R. R. Boutaba, and Y. Iraqi (2005), "Adaptive Packet Video Streaming over IP Networks: A Cross-Layer Approach,” IEEE J. on Selected Areas in Communications, vol. 23 , no. 2 .

[28] R. Kusching, I. Kofler, and H. Hellwanger, (2010) "Improving Internet Video Streaming Performance by Parallel TCP-based Request Response Streams", Proc. of IEEE CCNC 2010. 


\section{Authors Short Biography}

Ghaida A. AL-Suhail is an Assistant Professor at the University of Basrah, Computer Engineering Department, Basrah, IRAQ. She received the PhD Degree in Electrical Engineering from the University of Basrah, in 2007. Her current research interests include wireless networks and communications, mobile multimedia, image processing, Internet Protocols, Cross-layer design, Quality of Service, modelling and Simulation, and mobile computing. She has been a Fulbright Scholar 2011 at the Michigan State University (MSU) in USA and Endeavour Fellowship 2009 Scholar at the Australian National University (ANU), RSISE, in Australia. She published several papers in International Journals and Conferences like JMM, IJMCMC, IJCNC, WSEAS'06, ICARCV'06, MoMM08/2009, ICSPCS7/8/9/2010 (Australia and USA), WiMo2011 (Turkey) and EPC-IQ01 and CCIT2012 (IRAQ).

Sarah A. Subber received B.E. Degree in Computer Engineering from the University of Basrah, in 2004. She is currently MSc. Student at the Computer Engineering Department, University of Basrah, IRAQ. Her research interests include video streaming, wireless channels, network performance simulations, network simulators, TCP/UDP protocols and cellular networks.
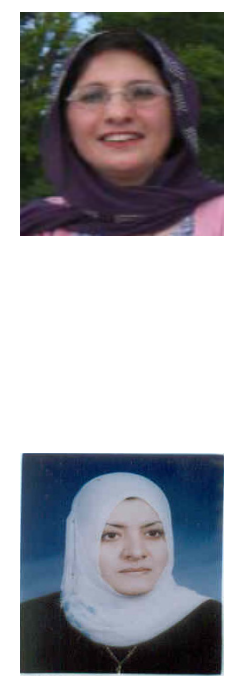